\title{
Un dispositivo de reflexión sobre las prácticas y las relaciones con el saber. «La experiencia de un docente en una escuela técnica neuquina»
}

\section{A device for reflecting on teaching practices when revising relationships with knowledge. The experience of a teacher at a technical school in the province of Neuquén, Argentina}

\author{
Bardelli, Noemí; De Vega, Micaela
}

\author{
Noemí Bardelli Sobre la autora \\ noemibardelli@gmail.com \\ Consejo Nacional de Investigaciones Científicas \\ (IPEHCS-CONICET), Argentina \\ Micaela De Vega Sobre la autora \\ micaeladevega@gmail.com \\ Universidad Nacional del Comahue (UNCO), \\ Argentina
}

\section{Itinerarios educativos \\ Universidad Nacional del Litoral, Argentina \\ ISSN: 1850-3853 \\ ISSN-e: 2362-5554 \\ Periodicidad: Anual \\ vol. 1, núm. 14, 2021 \\ revistadelindi@fhuc.unl.edu.ar}

Recepción: 03 Noviembre 2020

Aprobación: 05 Julio 2021

URL: https://doi.org/10.14409/ie.2021.14.e0007

\section{(1) (2)}

Esta obra está bajo una Licencia Creative Commons AtribuciónNoComercial-CompartirIgual 4.0 Internacional.
Resumen: Se presenta la experiencia de reflexión sobre las prácticas de enseñanza de un docente de escuela secundaria técnica agropecuaria de la provincia de Neuquén, que tuvo lugar durante el año 2019, en el marco del programa de investigaciónacción desarrollado por el Equipo de investigación C-127 de la Universidad Nacional del Comahue. El dispositivo que se generó en dicho programa intentó movilizar los saberes del docente en cuestión y lo desafió a la construcción de sus problemas didácticos formando parte de un ciclo de reflexión-acción que instaló nuevas preguntas y enigmas sobre las relaciones con el saber que propiciaron un corrimiento de las figuras del aprender en la búsqueda de nuevas prácticas de enseñanza. Se reconstruye el proceso del docente reconociendo situaciones que él mismo entendía como problemáticas incurriendo luego en revisión de las decisiones didácticas en pos de repensar las propuestas de enseñanza.

Palabras clave: reflexión, relación con el saber, prácticas de enseñanza, dispositivo escolar.

Abstract: This work addresses a reflection on the teaching practices of a teacher at an agricultural and technical high school in the province of Neuquen. These practices were introduced in 2019 in the framework of an action-research program developed by the Research Team C- 127 of the National University of Comahue. The program included a device that was intended to activate teachers' knowledge and challenge them to a new construction of didactic problems. This construction had to be part of a cycle of reflection-action that introduced new questions and inquiries into the relationships with knowledge that fostered a shift in the representations of learning when seeking new teaching practices. The undergone process is reconstructed, recognizing situations the teacher considered problematic and undertaking a review of didactic decisions in order to rethink teaching proposals.

Keywords: reflection, relationship with knowledge, teaching practices, school device. 


\section{Prácticas de enseñanza que construyen saberes}

Si hay algo que perdura en la Escuela, a pesar de todo, es la relación del sujeto con el saber, que el papel del profesor debe ser capaz de animar. ¿Existe aún la posibilidad de introducir al sujeto en una relación vital con el saber? lo que queda de la Escuela ¿no es acaso la posibilidad, una y otra vez nueva, de transformar los objetos del saber en objetos de deseo, en cuerpos eróticos? (Recalcati, 2016: 7)

Frente a la pregunta ¿cómo las prácticas de enseñanza en clave emancipadoras, reflexivas y creativas posibilitan los procesos de construcción de los saberes docentes? nos resulta pertinente recuperar cierta tradición en las pedagogías latinoamericanas que ponen de manifiesto otras relaciones de los/as docentes con el saber pedagógico (Kohan, 2013). Esas relaciones con el saber pedagógico nos invitan a poner en análisis la capacidad de invención en el trabajo de enseñar, reconociendo la existencia de saberes que siguen siendo nominados y no saberes dominados, en términos del trabajo docente. Esto implica romper con ciertas estructuras hegemónicas acerca de cómo los/as docentes producen saberes en situación recuperando los sentidos pedagógicos de la tarea didáctica.

Existen diversas formas de llamar a los saberes que se tejen en la misma experiencia educativa. Algunos autores se refieren a éstos como saberes de oficio, saberes de referencia, saberes prácticos, saberes del trabajo, saberes ordinarios (De Vega y Bardelli, 2020). Más allá de los sentidos donde se asienta el carácter experiencial, en todos los casos estos saberes «constituyen una herramienta para la acción y es la práctica su fuente de referencia y legitimación. A través de distintos procedimientos (sistematización, crítica, confrontación, conceptualización) este tipo de saber transforma el «arte» o la «experiencia» en algo que puede enseñarse y aprenderse» (Alliaud en Terigi, 2013:87). Según Ferry (1991, en Alliaud, 2004) estos saberes surgen de la implicación en los procesos de formación cuyo carácter inacabado e incompleto da sentido a la construcción de propios trayectos, donde se interiorizan:

Modelos de enseñanza (Lortie, 1975); adquieren saberes y reglas de acción (Terhart, 1987), pautas de comportamiento (Gimeno Sacristán, 1992); construyen esquemas sobre la vida escolar (Contreras Domingo, 1987); se forman creencias firmes y perdurables (Jackson, 1999), imágenes sobre los docentes y su trabajo (Rockwell, 1985), teorías, creencias supuestos y valores sobre la naturaleza del quehacer educativo (Pérez Gómez, 1997) (Alliaud, 2004: 2)

Asimismo, el no saber en la docencia se presenta como la posibilidad de encontrar-se en las experiencias escolares que se viven en el propio ejercicio del oficio (Alliaud y Antelo, 2009). Estas son las que suscitan nuevas relaciones entre el saber pedagógico y la acción educativa, profundizando y complejizando sus posibles interrelaciones. Partimos de reconocer que «el saber pedagógico existente parece no ser suficiente para dar respuesta a los problemas del presente, de nuestro sistema educativo y de las prácticas docentes» (Terigi, 2013:9). Justamente esto nos permite detenernos a pensar: ¿qué nuevas relaciones con los saberes docentes se construyen en las clases a propósito de la resolución local de problemas inesperados y emergentes a la hora de seguir enseñando? y ¿qué saberes objetivados propios de la formación inicial se resignifican a la luz de la enseñanza? 
Si entendemos a la enseñanza desde sus intencionalidades político y pedagógicas, son las mismas prácticas y las relaciones con el saber que allí se promueven las que propician una forma de saber que abre nuevas posibilidades a la propia tarea docente incidiendo en la invención de variadas y creativas oportunidades de aprendizaje. Será necesario repensar que todo saber es incompleto e inacabado, siempre obra por hacer; quizás por eso en nuestros oficios se constate siempre que los saberes que nos ofrecieron en la formación son insuficientes, inadaptados e inapropiados (Frigerio, Korinfeld y Rodriguez, 2018). Toda relación con el saber nunca es definitiva haciendo entrar al sujeto en conflicto (Kalali en Vercellino, 2018). Será necesario reflexionar, para volvernos precursores de nuestros saberes acerca de la enseñanza, de modo tal que en algún momento podamos reconocer las relaciones que construimos con los saberes transformando nuestras prácticas, subjetivando el oficio y reinventando la identidad docente.

\section{Acerca de un dispositivo de reflexión y las posibilidades de repensar las relaciones con el saber}

Según Alliaud (2010) se experimentan transiciones en el aprender a enseñar que adquieren el sentido de obras de enseñanza visibles, contempladas, admiradas y criticadas por otros/as con los que se comparte el oficio, siempre y cuando la enseñanza sea considerada una tarea colectivamente construida y discutida dentro de las instituciones educativas.

Por ello, pensamos a la reflexión como un proceso cíclico de carácter colectivo, plural y participativo que parte de recuperar las situaciones vividas en las prácticas por los/as docentes como problemas que incomodan, movilizan y apremian a su resolución. Entendemos a los problemas como construcciones intencionales que permiten generar acciones de transformación y mejora de las prácticas desde una perspectiva didáctica (De Vega y Bardelli, 2020). En este marco, la reflexión sobre los saberes necesarios para poder enseñar se habilita en dos sentidos. Por un lado, poner los propios saberes docente en obra, en situación de transformación y por otro lado, reconocer en esos saberes las nuevas posibilidades que se abren para obrar interviniendo en las prácticas a partir de dispositivos de reflexión, a la vez personal y colectivos, sobre cómo se ve el mundo desde una sala de aula (Larrosa, 2019).

En tal sentido es posible advertir, que para dar lugar a la reflexión es propicio habilitar el espacio que contenga dicho proceso. Denominamos a esa creación como dispositivo, considerando que estos son «... artificios creados para producir algo, para generar procesos en los sujetos, para ordenar» (Greco, 2020:8). Los mismos son necesarios de ser revisados, reflexionados ya que su carácter relacional y estratégico posibilitan abrir espacios y prácticas de negociación donde la subjetividad se pone en juego (Vercellino, 2020a). El dispositivo será el conjunto articulado y coordinado de tiempos, espacios, acciones y tareas, que se dan en lugares particulares a la vez que evidencian posiciones en una trama siempre dispuesta de relaciones que juegan con reglas que propician el funcionamiento de algún aspecto de las prácticas. Es necesario que tal dispositivo contenga hacia su interior acuerdos en aspectos teóricos, lo que impactará en las posiciones de los/ as docentes requiriéndose consensos en las acciones y modos de hacer. 
Desde el punto de vista epistemológico y metodológico, tomamos las reflexiones de Agamben (2011) quien concibe a un dispositivo como heterogéneo, como una red entre discursos, marcos normativos e instituciones. $\mathrm{Al}$ decir del autor, el dispositivo posee en sí mismo una «... función estratégica concreta, que siempre está inscrita en una relación de poder y como tal, resulta del cruzamiento de relaciones de poder y de saber» (Agamben, 2011:250)

A continuación, compartimos en el marco del Proyecto de Investigación C-127 «Escuela secundaria y trabajo en la provincia de Neuquén: dispositivos de formación, experiencias escolares y trayectorias juveniles» de FACE-UNCo una experiencia de reflexión sobre las prácticas basada en la investigación-acción desarrollada de marzo a noviembre del año 2019 conformada por un grupo de docentes-participantes pertenecientes a una escuela secundaria provincial de Educación Técnica Agropecuaria de Neuquén. Se inició el trabajo reconociendo necesidades y centros de interés de los/as docentes a través de actividades que permitieron desde las narrativas reconocer situaciones del trabajo pedagógico para identificar problemas didácticos en las prácticas. Luego se buscó orientar la construcción de actuaciones o estrategias didácticas de mejora o cambio para que los/as docentes implementaran en sus clases. Finalmente, nos centramos en valorar las propuestas implementadas en las clases para seguir profundizando la reflexión en y sobre la acción analizando y comparando lo sucedido en las aulas como experiencia buscando profundizar la reflexión, habilitando nuevas preguntas y visibilizando otros problemas didácticos para intervenir sobre las prácticas de forma recursiva a lo largo de todo el dispositivo.

Aquello que movilizó el trabajo en el dispositivo se centró en la pregunta en relación a ¿qué situaciones de las prácticas de enseñanza requieren una revisión y evaluación de estas? Encontramos que la posibilidad de conformar comunidades docentes que se piensen desde una reflexión sobre sus propias prácticas, y que a la vez conformen reflexiones colaborativas y representativas del conjunto, genera además de oportunidades de pensar y hacer con otros/as, habilita el lugar a la construcción de los problemas didácticos a partir de la discusión entre forma y contenido.

En este sentido, el recorrido de la reflexión se da a partir del encuentro con el problema. Asimismo, tal encuentro es a la vez el encuentro con el otro/a que, desde una perspectiva colaborativa, se aprende desde y en la reflexión al poner en tensión el origen o el motor de determinados problemas comunes en torno a la enseñanza. El encuentro con el problema no es simple, y no es una sucesión de pasos tecnocráticos que implican reconocer-reflexionar-aplicar, sino que éste se sumerge en una búsqueda más profunda de comprender en sí lo que motoriza a la enseñanza como una práctica sobre otros/as.

\section{La experiencia de un docente y sus relaciones con el saber}

A continuación, nos proponemos reconstruir el proceso reflexivo de uno de los docentes participantes como un análisis de caso que permite evidenciar un corrimiento de la figura del aprender (Charlot, 2006) y un proceso de construcción de nuevas relaciones con los saberes docentes. Motiva la elección de esta experiencia el reconocimiento de ciertos cambios que el docente 
experimentó a partir de la revisión de sus prácticas y de la reformulación de su propuesta didáctica a pesar de las dificultades a la adherencia al dispositivo.

\section{Situación inicial: relaciones con el saber como punto de partida}

Partimos de considerar que «el saber es una relación, un producto y un resultado. Relación del sujeto conocedor con su mundo, producido por la interacción entre el sujeto y su mundo, resultado de esta interacción» (Charlot, 2006:101) para comprender las relaciones que los docentes construyen con los saberes tomando distancia de la pretensión de hacer clasificable el saber del que los otros disponen por tipologías o características.

¿Qué situaciones trajo el docente al dispositivo en los primeros encuentros? y ¿qué preocupaciones, malestares e incomodidades expresó? Las situaciones que fueron analizadas por parte del docente provenían de su experiencia con un grupo de primer año en el marco de la materia Físico-Química. Entre ellas, en primer lugar, reconocemos experiencias y escenas que remiten a la centralidad por el contenido escolar a partir de estrategias didácticas basadas en el dictado de conceptos y definiciones. De ello se desprendían dificultades en los/as estudiantes respecto del no seguimiento a esa dinámica de trabajo propuesta que demandaba mera reproducción y copia textual. Como otra de las estrategias utilizadas se encontraba el trabajo con un material didáctico, un cuadernillo elaborado por el departamento del área que había sido heredado de otros docentes. El trabajo con el mismo duraba todo el año en función de lo planificado en el programa articulando teoría y práctica. De esto se reconocen señales de la figura del aprender que remiten a objetos que portan saberes promoviendo una relación epistémica denominada objetivación-denominación (Charlot, 2006). La centralidad de esta relación se evidencia en la necesidad de incorporar cosas a través de categorías y conceptos en tanto contenidos intelectuales que puedan ser nombrados y adquiridos. Aprender desde esta mirada radicará en pasar de la no posesión a la posesión del objeto que depende de su enunciación entendiendo que «El saber puede ser enunciado sin que sea evocado el proceso de aprendizaje» (Charlot, 2006:112)

En segundo lugar, reparamos en la mirada del docente sobre los/as estudiantes sesgada por preconceptos que remiten a apatía por la propuesta de enseñanza, y a una descripción infantil que se contraponía al estudiante que es esperado en la escuela secundaria. Esto último, a entender del docente, generaba una dependencia, en términos de afectividad, demostración, valoración y cercanía en el vínculo pedagógico. Esta demanda continua generaba incomodidad no pudiendo ser vista como un componente fundamental en la relación pedagógica. En consecuencia encontramos que se pone en juego las figuras del aprender vinculadas al dominio de relación de ambos, tanto de estudiantes como del docente, dejando una clara evidencia de un vacío en su formación pedagógica.

En tercer lugar, encontramos dudas respecto a una autoridad pedagógica mal entendida por el docente. A decir del docente interpretaba que si cedía a la demanda afectiva de los/as estudiantes se generaría una baja en su autoridad pedagógica. Lejos de pensar a la autoridad como una relación, sus acciones en el aula remiten a crear distancia de los/as estudiantes llamándolos por sus apellidos, regulando la ocupación de los espacios en la clase y llevando adelante prácticas 
vinculadas al control y visado del trabajo escolar. En consecuencia, el trabajo con este docente intentó abordar una concepción de autoridad en la que ésta no sea entendida como propiedad de quien ejerce poder sino quien funda un espacio, un proceso de formación y crea las condiciones para que ese proceso se despliegue (Arendt en Greco, 2020)

\section{Avanzando en la reflexión. ¿Qué problemas didácticos se construyeron?}

En los sucesivos encuentros que se generaron en el dispositivo el docente fue planteándose preguntas que se encontraban posicionadas en el afuera y en el hacer de otros/as. Surgieron así planteos como ¿qué necesitan mis estudiantes para aprender?

Encontramos como problema didáctico la preponderancia del contenido escolar. Esto remitió a la insistencia de una supuesta superioridad de ciertos conocimientos del docente a la hora de enseñar, imponiéndose la preocupación exclusiva del contenido disciplinar por sobre la forma de la enseñanza. De este modo el proceso de reflexión se centró en la revisión de lo metodológico, a partir de construir nuevos recursos de enseñanza, y generar un corrimiento de esa posición de objetivación-denominación para poner en valor el proceso de aprendizaje. Esto propició una toma de distancia a la vez, de una concepción de evaluación como amenaza y control. En tal sentido, estas prácticas y revisiones permitieron dar lugar a pensar las posibles articulaciones entre las lógicas disciplinares y metodológicas.

Como otro problema didáctico se presentó la necesidad de organizar las clases surgiendo la pregunta respecto a ¿cómo organizar el tiempo de la clase en función de sus momentos y las intencionalidades de cada uno de ellos? Esto derivó en repensar el posicionamiento del docente y el trabajo de enseñar, específicamente con relación a las tareas de planificación que demandaban tiempo, creatividad, flexibilidad ante la programación anual y la disponibilidad a realizar ajustes permanentes en función de la reflexión de su propia práctica. Reconocemos que el dispositivo en sus orígenes no pretendía ser una instancia formativa para quienes transitaban en él, pero disociar la reflexión de la formación es imposible siendo esta una posibilidad para revisar las relaciones de los/as docentes con el saber. A través de la construcción de este problema didáctico el docente, por añadidura, mostró movimientos como aprendiente de saberes propios de la enseñanza de los que carecía en su formación inicial (ingeniería agrónoma). En palabras de Charlot (2006) la figura del aprender que se evidenció en el proceso de este docente remite al pasaje de la denominación al dominio de la actividad de enseñar. De manera explícita reconocemos que en los distintos ensayos didácticos que el docente realizó en el tiempo para promover cambios y mejoras en sus propuestas de enseñanza, se generó una imbricación del yo que posibilitó un acercamiento a una posición reflexiva de su propia práctica (Charlot, 2006). 
En acción: ¿qué decisiones didácticas posibilitaron mejorar las prácticas de enseñanza?

Después de transitar la mitad del ciclo escolar y del dispositivo y con una dinámica de reflexión grupal, el docente fue tomando decisiones que surgían de las discusiones pedagógicas y didácticas entre pares. Entre las decisiones que se destacan encontramos la revisión de las estrategias de enseñanza centradas en la posibilidad de lo colaborativo y lo grupal. Esto implicó repensar la reorganización de sus clases en el tiempo a partir de definir intencionalidades, diseñar actividades y construir nuevos recursos que demandaban una posición activa y protagónica de los/as estudiantes. Estas decisiones motivaron el diseño de propuestas de enseñanza que llevaban a resignificar los espacios para el aprendizaje utilizando las distintas dimensiones que aportan los mismos, es decir, articulando lo vincular, lo epistémico y lo metodológico. Se plantearon distintos escenarios para la enseñanza como el laboratorio, el patio de la escuela y la chacra donde los/ las estudiantes experimentaban, hacían relevamientos, construían registros y tomaban contacto con los objetos de conocimiento. De ello se desprenden nuevas decisiones que el docente tomó en relación con la comunicación didáctica, sin perder de vista su preocupación por el cumplimiento de las exigencias que demanda la formación técnico-profesional centradas principalmente en el uso técnico del vocabulario y el respeto por las actividades del agro. Esto incidió en los vínculos que el docente construyó con los/as estudiantes generando cercanía y mayor encuentro e incluso entendimiento entre ellos.

Asimismo, estas decisiones impactan en los sentidos atribuidos a la transmisión de saberes en el trabajo de enseñar considerando nuevas priorizaciones de los contenidos en su orden, relevancia y profundidad teniendo en cuenta la trayectoria escolar de los/as estudiantes. En este marco, las decisiones permitieron dar lugar a pensar nuevas articulaciones que habilitaron preguntas respecto a su posición como docente, sus concepciones de enseñanza y aprendizaje y las relaciones con el saber que construía.

\section{A modo de conclusión: ¿Es posible enseñar sin construir relaciones con el saber?}

Partimos de esta pregunta dado que nos interesa detenernos en estas últimas líneas a repensar cómo «... el saber es una relación, un producto y un resultado. Relación del sujeto conocedor con su mundo, producido por la interacción entre el sujeto y su mundo, resultado de esta interacción » (Charlot, 2006:101).

Instalar en la reflexión la inquietud por la transmisión abre las posibilidades de comprender que la educación tiene que ver con transmitir relaciones con el mundo, en palabras de Larrosa (2020) entregarlo a los/las que vienen para preservar lo que hoy tenemos haciendo de esa experiencia un tiempo renovado. En este sentido, reflexionar sobre las prácticas de enseñanza refleja que en cada propuesta didáctica los/as docentes reconstruyen sus propias relaciones con el saber. Al llevar adelante las clases cada docente invita a otros, (estudiantes) a pensar sus propias relaciones con el saber presentando el mundo, abriéndolo, trayéndolo al aula, dándole sentido, prestándole atención, describiéndolo y estudiándolo. Hacer que un pedacito del mundo sea un contenido escolar está 
lejos de pensar que las relaciones con el saber son en términos lineales o causales, sino que generan un movimiento en el/la otro/a abriendo nuevas posibilidades de estar en el mundo, es decir, renovando la propia inscripción en él a partir de la atención, la sensibilidad, la precisión y la reflexión. El mismo autor propone pensar que invitar como docentes a otros/as a una relación con el saber es una práctica de cuidado que motiva al atrévete a saber.

En este sentido, el dispositivo desarrollado intentó movilizar los saberes del docente en cuestión y lo desafió a un atrévete a construir tus problemas didácticos iniciando así un ciclo de reflexión-acción que instaló nuevas preguntas y enigmas sobre las relaciones con el saber que propiciaron un corrimiento de las figuras del aprender en la búsqueda de nuevas prácticas de enseñanza. La posibilidad de modificar las relaciones con el saber también incidió en la percepción que él tenía de los estudiantes considerando sus posibilidades de aprender y el modo en el que éstos se posicionaron frente al saber escolar en sus clases.

Partiendo de considerar que el saber se ejerce siempre en interacción, incluso colectivamente, reconocemos que la potencia del dispositivo estuvo centrada en los saberes colectivos construidos con pares docentes con los que se compartían experiencias didácticas, una historia institucional y la situacionalidad del aprendizaje escolar generando una alteración así de los propios saberes y afectando los modos de ver, entender y hacer. Es por ello que se reconoce en esta experiencia que «Todo saber resulta en cada sujeto, de un cruce infinito de variables, modos singulares de tramitar y modos colectivos e individuales de significar que se subjetivan» (Frigerio y Diker, 2010:9)

Para concluir, retomando la experiencia en cuestión, desde el dispositivo también se propuso motivar la transformación de ciertos aspectos del trabajo de enseñar, como una práctica tradicional, apostando al saber que se produce en la reflexión con otros/as. Allí circuló lo que se sabe y lo que no se sabe, lo que se teme y lo que no, lo dado y lo esperado. ¿Es posible encontrar en la revisión de las relaciones con el saber que construyen los/as docentes claves para la mejora de la enseñanza?, ¿encontrarán las instituciones escolares los modos de sostener dispositivos de reflexión que movilicen interpelando a los/as docentes y sus prácticas?

\section{Referencias}

Agamben, G. (2011) «¿Qué es un dispositivo?». Revista Sociológica, 26, (79): 249-264

Alliaud, A. (2004). «La experiencia escolar de maestros inexpertos. Biografías, trayectorias y práctica profesional». Revista Iberoamericana de Educación $34(1): 1-11$.

Alliaud, A. (2010). «Experiencia, saber y formación». Revista de educación (1):141-57.

Alliaud, A. \& Antelo, E. (2009). Los gajes del oficio: enseñanza, pedagogía y formación. Buenos Aires: Aique.

Beillerot, J. (1998). La formación de formadores: entre la teoría y la práctica. Buenos Aires: Novedades Educativas.

Charlot, B. (2006). La relación con el saber. Formación de maestros y profesores, educación y globalización Cuestiones para la educación de hoy. Montevideo: Ediciones Trilce.

De Vega, M. y Bardelli N. (2020). «Clase 3: Saberes docente "en obra". Intersecciones políticas en la configuración del trabajo docente y la autoridad pedagógica. En: 
Itinerario formativo La enseñanza en escena. Lo urgente, lo necesario y lo relevante en el campo pedagógico y didáctico. Secretaría de educación. Escuela "Marina Vilte" Formación Pedagógica y Sindical» Disponible en: https://educacion.ctera.org.ar /clase-3-curso-de-la-ensenanza-en-escena/

Ferry, G. (1991). El trayecto de la formación: los enseñantes entre la teoría y la práctica. México: Paidós.

Frigerio, G. \& Diker, G. (2010). Educar: saberes alterados. Buenos Aires: Del Estante.

Frigerio, G, Korinfeld, D. y Rodriguez, C. (coords) (2018). Saberes de los umbrales: Los oficios del lazo. Buenos Aires: Novedades Educativas.

Greco, B. (2020). «Clase 6: "Sobre intervenciones institucionales y dispositivos en clave de inclusión» En: Curso Autoridad Pedagógica. Intervenciones y dispositivos que "hacen" escuela. Buenos Aires: Fundación de Sociedades Complejas.

Kohan, W. (2013). El maestro inventor: Simón Rodríguez. Buenos Aires: Miño y Dávila.

Larrosa, J. (2019). Esperando no se sabe qué. Sobre el oficio de profesor. Buenos Aires: Noveduc.

Larrosa, J. (2020). «La materialidad del oficio docente» En: Congreso internacional de Educación Almirante Brown, Secretaría de Educación Almirante Brown: Provincia de Buenos Aires. Disponible en: https://www.youtube.com/watch? $\mathrm{v}=\mathrm{RrCBnMFR} 6 \mathrm{cA} \& \mathrm{t}=7 \mathrm{~s}$ escrita)

Recalcati, M. (2016). La hora de clase: por una erótica de la enseñanza. Vol. 504. Anagrama. Disponible en: https://interabide.wordpress.com/2017/03/22/la-ho ra-de-clase-por-una-erotica-de-la-ensenanza-massimo-recalcati/

Terigi, F. (2013). «Saberes docentes: qué debe saber un docente y por qué». VIII Foro Latinoamericano de Educación. Buenos Aires: Santillana. Disponible en: https:// www.fundacionsantillana.com/PDFs/8vo_foro.pdf

Vercellino, S. (2018). La escuela y los (des)encuentros con el saber. Argentina: Editorial UNRN.

Vercellino, S. (2020a). «Clase 1: La institución educativa en tiempos de (as)dispo» Curso: Enseñar y aprender en la incertidumbre: Problematizaciones necesarias. Universidad Nacional de Río Negro.

Vercellino, S. (2020b). «Clase 3: El Saber». Curso: El Sujeto y la relación con el saber. CURZA UNCO.

\section{Notas}

[1] Licenciada y Profesora en Ciencias de la Educación (F.F y L, UBA). Es Magister en Psicología Cognitiva y Aprendizaje (FLACSO). Se desempeña como docente de Nivel Superior y actualmente desarrolla tareas de investigación en el proyecto «Escuela secundaria y trabajo en la provincia de Neuquén: dispositivos de formación, experiencias escolares y trayectorias juveniles» en la UNCo. Participa de distintos espacios para la Formación de la Escuela «Carlos Fuentealba» de ATEN Provincial. Doctoranda en Educación en la UBA.noemibardelli@gmail.com

[2] Profesora en Ciencias de la Educación FACE, UNCo). Es especialista en Asesoramiento Educacional por la UNCo. Se desempeña como profesora regular en Planeamiento y Administración de la Educación en la FACE, UNCo. Es formadora de formadores en profesorados de educación primaria e inicial de Neuquén. Integrante del equipo de Proyecto C127 de la FACE, UNCo, «Escuela secundaria y trabajo en la provincia de Neuquén: dispositivos de formación, experiencias escolares y trayectorias juveniles».micaeladevega@gmail.com 


\section{Notas de autor}

Sobre la autora

Licenciada y Profesora en Ciencias de la Educación (F.F y L, UBA). Es Magister en Psicología Cognitiva y Aprendizaje (FLACSO). Se desempeña como docente de Nivel Superior y actualmente desarrolla tareas de investigación en el proyecto «Escuela secundaria y trabajo en la provincia de Neuquén: dispositivos de formación, experiencias escolares y trayectorias juveniles» en la UNCo. Participa de distintos espacios para la Formación de la Escuela «Carlos Fuentealba» de ATEN Provincial. Doctoranda en Educación en la UBA.

Sobre la autora

Profesora en Ciencias de la Educación FACE, UNCo). Es especialista en Asesoramiento Educacional por la UNCo. Se desempeña como profesora regular en Planeamiento y Administración de la Educación en la FACE, UNCo. Es formadora de formadores en profesorados de educación primaria e inicial de Neuquén. Integrante del equipo de Proyecto C127 de la FACE, UNCo, «Escuela secundaria y trabajo en la provincia de Neuquén: dispositivos de formación, experiencias escolares y trayectorias juveniles». 